\title{
Spectroscopic Measurement of Secondary Arc Plasma on Solar Array
}

\author{
By Takayuki OSE, Kazuhiro TOYODA, Hirokazu MASUI, Mengu CHO
}

Laboratory of Spacecraft Environment Interaction Engineering, Kyushu Institute of Technology, Kitakyushu, Japan

\author{
(Received May 2nd, 2008)
}

\begin{abstract}
As the power level of Geostationary satellites increases, discharge phenomena on solar array are becoming serious threat to safe operation. Arcs on solar array can short-circuit the satellite circuit, decrease the satellite power, and then cause the satellite permanent failure. To prevent the failure caused by charging and arcing, it is necessary to investigate the mechanism of satellite charging and arcing phenomenon. The purpose of this paper is to investigate the occurrence condition of a secondary arc by measuring arc plasma characteristics in ground test. We measured the arc plasma temperature and identified the materials emitted using spectrometer at arbitrary time during arc occurring. We investigated the difference of secondary arcs occurrence condition during secondary arcs. From the spectroscopic measurement results, we found that it was necessary for shifting to the secondary arc that the metallic vapor same as the cathode material was emitted. In case of primary arc (PA) dimension changes, the probability of secondary arc and TSA occurrence became high. And plasma temperature was not affected by PA dimension, however the metallic vapor emission of silver was greatly affected. Thus, secondary arc occurrence greatly depends on metallic vapor emission from cathode.
\end{abstract}

Key Words: Secondary Arc, Spectroscopy, Temperature

\section{Introduction}

In geostationary earth orbit (GEO), thin hydrogen plasma exists at density of $10^{6} \mathrm{~m}^{-3}$. Substorm phenomenon sometimes occurs due to earth magnetic field disturbance according to the change of the solar wind. High energy electron flux of tens of $\mathrm{keV}$ flows into the satellite when the substorm occurs. In such an environment, arcs occur on the solar array and threaten the safe operation, as the bus power level of satellite increases. The arcs due to interaction between space plasma environment and solar array are serious problem for satellites ${ }^{1)}$. Therefore, it is important to investigate the mechanism of the charging and arcing phenomena of the satellite.

When the satellite is charged, the coverglass potential can be higher than the interconnector potential which is equal to the spacecraft ground because of the difference in secondary emission yield. This is called the inverted potential gradient condition. When the inverted potential gradient is formed, the high electric fields are formed at the triple junctions. The electrons are emitted from the surface of the metal by the electron field emission. The secondary electron is emitted there by colliding with side edge of the cover glass. Then, the electric field near the triple junction is enhanced further. As the field emission current increases, the gas desorption increases at side edge of the cover glass and forms the gas layer. The arc occurs by ionization in the gas ${ }^{2)}$. This arc phenomenon is called primary arc. In addition, the solar array cell can be short-circuited with the conductive substrate through the arc plasma formed by primary arc and the arcs cause short-circuit between adjacent strings with operational voltage (Fig. 1). This arc phenomenon is generally called secondary arc. If secondary arc occurs, the power cannot be supplied to the onboard instrument of satellite because the output power of the solar array circuit is lost by short-circuit between a positive electrode and a negative electrode.

The kind of arc (PA) is classified by the current waveforms flowing between adjacent strings as shown in Fig. 2. Primary arc is an arc phenomenon that the charge stored on the cover glass flows into the arc spot through the arc plasma. This current that flows into electrode is called as a blow-off current. If there is no secondary arc after a blow-off current finishes, the arc is called the non-sustained arc (NSA). The temporary sustained arc (TSA) and the permanent sustained arc (PSA) are the arc phenomena that the current generated by solar cells flows into cells of adjacent strings through the arc plasma and is maintained even after blow-off current ends.

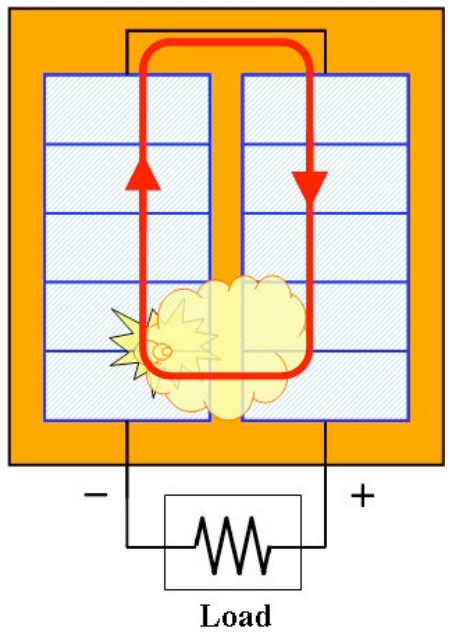

Fig. 1 Current path of secondary arc. 


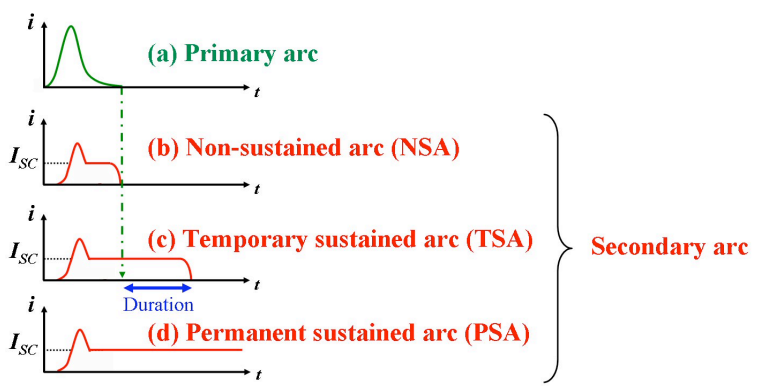

Fig. 2 Definition of arc current based on current waveform.

This current flowing into the cells of adjacent strings is called as an arc current. Thus, arc plasma might be maintained or not even if the trigger arcs are same. However, the condition of maintained arc plasma which is occurrence condition of secondary arc is not well-known.

Our purpose of this study is to find the occurrence condition of secondary arcs causing satellite failure by measuring the plasma temperature and the emission metallic vapors by spectroscopy. In this paper, we measured the emission spectrum of arc discharge in arbitrary timing. We calculated the arc plasma temperature associated with arc plasma conductivity, and identified the kind of material emitted from solar array due to arcs. We considered the difference in the property of the arc plasma between NSA and TSA during the secondary arc.

\section{Experimental Setup}

\subsection{Solar Array Coupons}

We used solar array coupons designed for Japanese satellite ETS-VIII ${ }^{3)}$, and composed of $\mathrm{Si}$ cells (70x35 $\mathrm{mm}$ ). This coupon is used to investigate the secondary arc inception between adjacent strings (Fig. 3). Test coupon has no scar on the surface. We biased 4 cells of adjacent strings (masked cells in Fig. 3), and examined the arc phenomena where arc occurs at between the different potential strings.

\subsection{Experimental Setup}

All experiments were performed in a vacuum chamber, which has $600 \mathrm{~mm}$ in diameter and $900 \mathrm{~mm}$ in length. The pressure in the chamber was less than $2 \times 10^{-4} \mathrm{~Pa}$ during experiment. Figure 4 shows the experimental circuit for arcs between strings. The electron beam gun (acceleration voltage $0 \sim 30 \mathrm{kV}$, electronic current densities $0 \sim 200 \quad \mu \mathrm{A}$ ) (ULVAC: RHEED) simulates the high energy electron that flows into the satellite at the substorm. Moreover, high voltage power supply $\mathrm{V}_{\text {bias }}(0 \sim 60 \mathrm{k} \mathrm{V}, 0 \sim 10 \mathrm{~mA})$ (Glassman: EW60R10) was used to simulate the potential of satellite sinking negatively during the substorm.

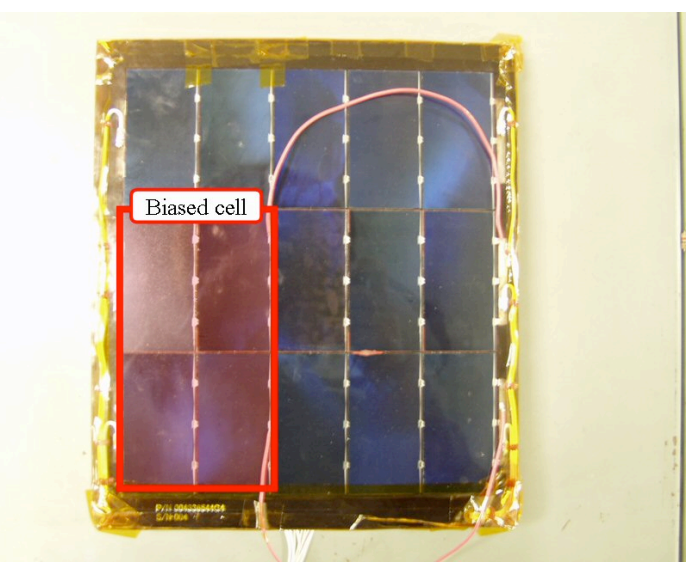

Fig. 3 Overview of test coupon.

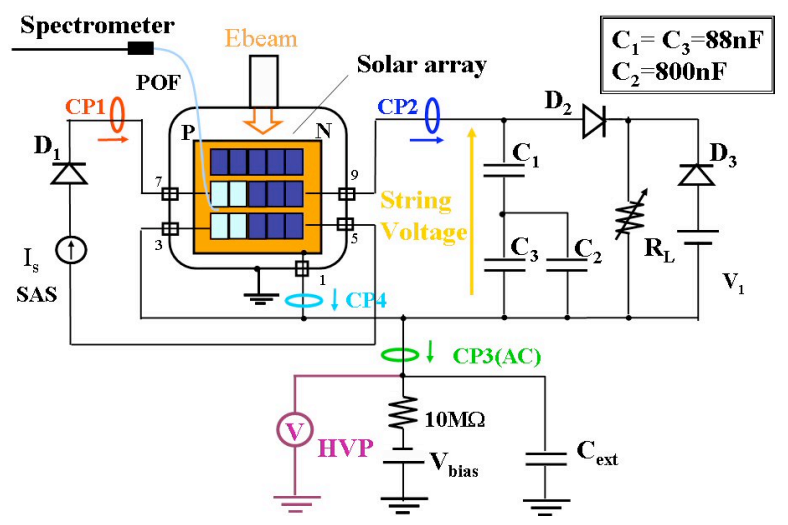

Fig. 4 Experimental circuit.

In addition, a solar array simulator SAS (Agilent: E4351B) was used to simulate the power generation of solar array and supplied to the resistance RL that simulated the load of the satellite. The voltage between the cells and the substrate (voltage between strings) was assumed to be $105 \mathrm{~V}$. The $\mathrm{C}_{\text {ext }}$ that simulated the charge stored in other cover glass was set $20 \mathrm{nF}$ because the solar array of an actual size could not be put in the chamber.

When an arc occurs, electrons are emitted from $\mathrm{C}_{\mathrm{ext}}$ and flow through the solar array, and then reach the chamber wall through the arc plasma, and flow to the ground. In this case, the current measured at CP3 in the experiment circuit is called a blow-off current. In the case of NSA, the SAS keeps supplying the current to RL after the blow-off current finished flowing. In the case of TSA, the current flows into the substrate and cannot flow to RL because RL is supplied with a voltage of V1 which simulates generated voltage by other solar array.

\subsection{Emission spectroscopy}

In this experiment, the spectroscopy was carried out using a spectrometer (Hamamatsu Photonics: PMA11 C8808-01). It has the measurement range of wavelength of $200 \mathrm{~nm} \sim 860 \mathrm{~nm}$, the minimum time resolution of $10 \mathrm{~ns}$, the wavelength resolution of $3.0 \mathrm{~nm}$. Moreover, a plastic optical fiber POF (Edmund: 53833-G) was attached to the detector to raise intensity from arc discharge.

The oscilloscope outputs the trigger signal to a delay pulse generator DPG (Stanford Research: DG-535) 
detecting a rising edge of a blow-off current when the arc occurs. The trigger signal is sent to the DPG and makes a TTL (Transistor Transistor Logic) and an ECL (Emitter Coupled Logic) signal to the spectrometer. The emission spectrum from arc is taken by the detector at time when ECL signal rises. Spectrum acquisition in arbitrary timing after the arc becomes possible by changing delay time (Delay) and pulse width (Gate) of the ECL signal.

The data base of NIST (National Institute of Standards and Technology) ${ }^{4)}$ was used to identify the measured spectrum. C2 swan band spectrum (420 nm 530 nm) with high reproducibility was used to calculate the temperature of plasma. C2 swan spectrum at from $2000 \mathrm{~K}$ to $7000 \mathrm{~K}$ is open to the public in BESP ( Boltzmann Equilibrium Spectrum Program) ${ }^{5)}$. Figure 5 shows C2 swan spectrum at $6000 \mathrm{~K}$. We paid attention to the area ratio of the first mountain to the second mountain. The correlation with the temperature and the area ratio is shown in Fig. 6. It was found that the temperature was represented by the area ratio using the regression curve of exponential.

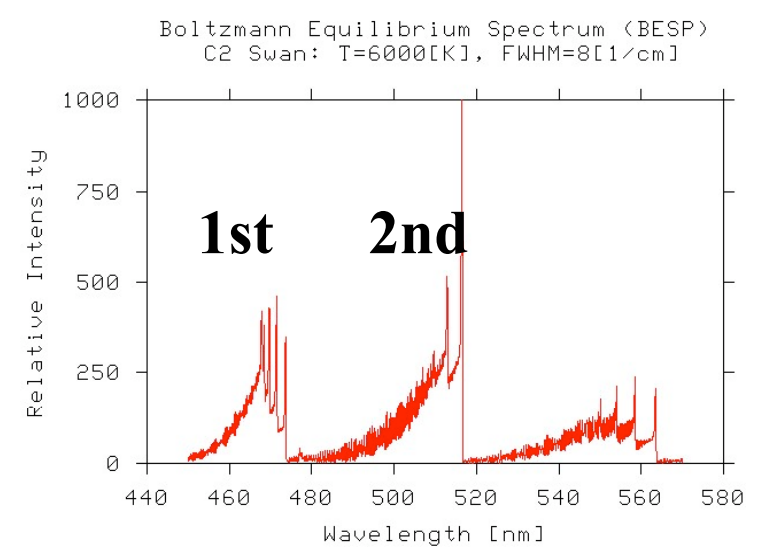

Fig. 5 C2 swan Spectrum in 6,000K (BESP) ${ }^{5)}$.

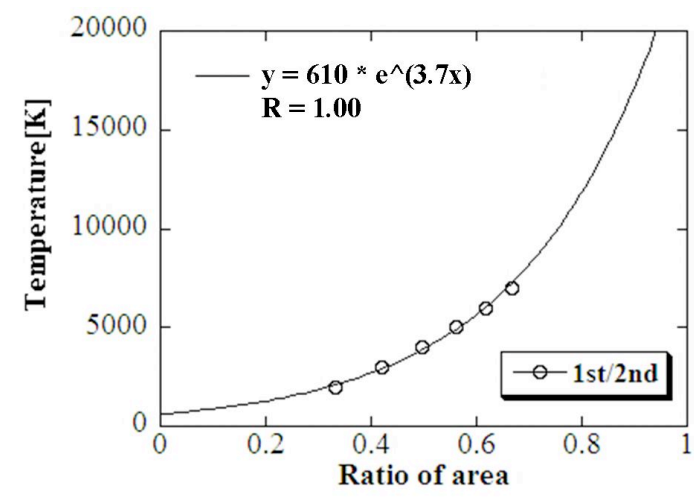

Fig. 6 Relation between ratio and temperature of area.

\section{Experimental Result and Discussion}

Table 1 shows the experimental parameter used in this experiment. The experiments was performed at about $2 \sim 8 \times 10^{-4} \mathrm{~Pa}$. The string voltage is potential difference between two adjacent solar array strings. The Is is the current supplied from SAS. The used electron beam current density was larger than that of space environment in order to cause adequate number of discharges in limited time.

\subsection{Experiment of Arcs at String Gap}

Time Variation of Arc Plasma Temperature for PA and TSA

This experiment was carried out by the parameter in Table 1 excluding the following parameters (Is=1.2 A, Gate:2.5 $\mu$ sec). Table 2 lists the number of arcs measured during this experiment. There was no NSA in this experiment. There are two types of TSA; TSA-Hot is that $t$ arcs occur at the high potential cell's electrode, TSA-Rtn is that arcs occur at the low potential cell's electrode.

Figure 7 shows plasma temperature distribution to time variation for PA and TSA. The time in a blow off current ending is defined as $0 \mu \mathrm{sec}$ (Time $=0 \quad \mu \mathrm{sec}$ ). As shown in Fig. 7, there is no difference in temperature distribution while a blow off current is flowing in PA and TSA.

Table 1 Experimental parameter

\begin{tabular}{|c|c|c|}
\hline \multicolumn{2}{|c|}{ Back Pressure } & $2 \times 10^{-4} \mathrm{~Pa} \sim 2 \times 10^{-3} \mathrm{~Pa}$ \\
\hline \multicolumn{2}{|c|}{$\mathrm{C}_{\mathrm{ext}}$} & $20 \mathrm{nF}, 30 \mathrm{nF}, 45 \mathrm{nF}, 65 \mathrm{nF}$ \\
\hline \multicolumn{2}{|l|}{$\mathrm{V}_{\mathrm{s}}$} & $70 \mathrm{~V}, 90 \mathrm{~V}, 105 \mathrm{~V}$ \\
\hline \multicolumn{2}{|l|}{$\mathrm{I}_{\mathrm{s}}$} & $\begin{array}{c}0.05 \mathrm{~A}, 0.1 \mathrm{~A}, 0.2 \mathrm{~A} \\
0.5 \mathrm{~A}, 1.2 \mathrm{~A}\end{array}$ \\
\hline \multicolumn{2}{|c|}{$\mathrm{V}_{\text {bias }}$} & $-7 \mathrm{kV}$ \\
\hline \multicolumn{2}{|c|}{ Electron Beam } & $7.2 \mathrm{keV} \sim 8 \mathrm{keV}, \sim 1 \mathrm{~mA} / \mathrm{m}^{2}$ \\
\hline \multirow{3}{*}{ Spectrometer } & Gate & $1 \mu \mathrm{sec}, 2.5 \mu \mathrm{sec}$ \\
\hline & Delay & $0 \sim 30 \mu \mathrm{sec}$ \\
\hline & Gain & 6 or 8 \\
\hline
\end{tabular}

Table 2 Number of arcs at string gap

\begin{tabular}{c|c|c|c|c}
\hline \hline Is [A] & Arcs at gap & PA & TSA-Hot & TSA-Rtn \\
\hline 1.2 & 10 & 9 & 18 & 22 \\
\hline \hline
\end{tabular}

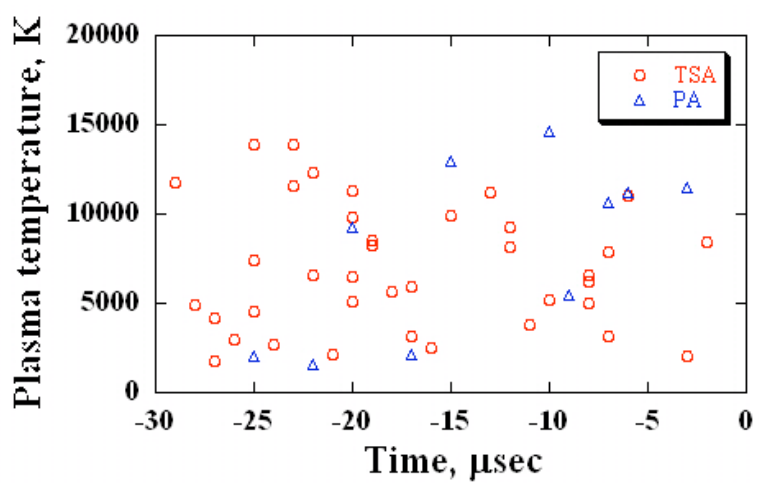

Fig. 7 Plasma temperature distribution as a function of time variation for PA and TSA.

Figure 8 shows that relation between arc plasma temperature and TSA duration before primary arc finished. As shown in Fig. 8, the TSA duration did not depend on the plasma temperature.

We examined the relative intensity of silver that was used for the material of cell's electrode. Figures 9 and 10 show 
the spectrum of PA and TSA. We found that silver metallic vapor (masked area in Fig. 10) was emitted more in case of TSA than that in case of PA.

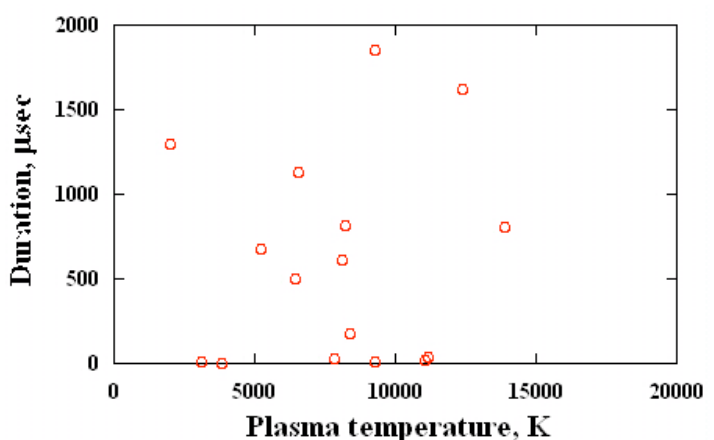

Fig. 8 Relation between arc plasma temperature and TSA duration.

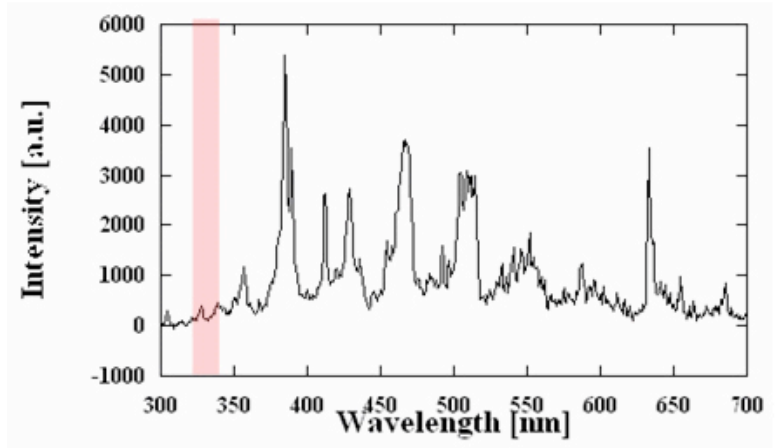

Fig.9 Arc spectrum of PA.

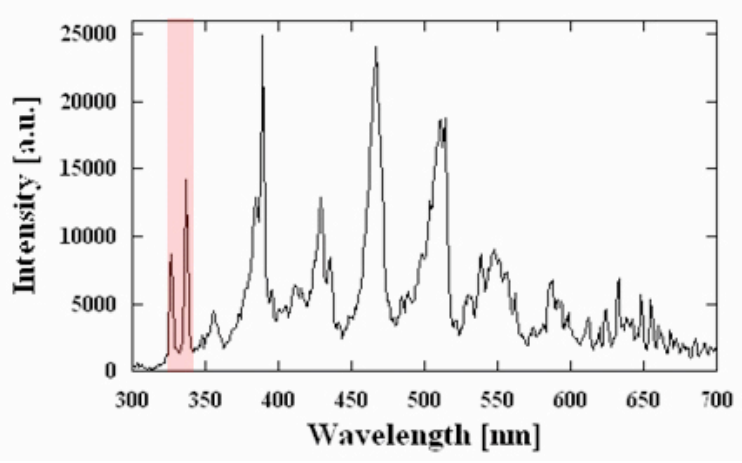

Fig. 10 Arc spectrum of TSA.

Relation between emitted metallic vapor and probability of TSA transition

The condition of shifting from PA to TSA was considered by focusing on relative intensity of spectrum of $\mathrm{Ag} \mathrm{I}$.

Figure 11 shows the relation between probability of shifting to TSA and relative intensity of $\mathrm{Ag} \mathrm{I}$ and first peak of $\mathrm{C} 2$ molecular. $\mathrm{C} 2$ molecular was measured reproducible at each spectrum. This figure shows that the probability of arc shifting to TSA was high if the amount of silver metallic vapor which composes the cell's electrode was emitted much.

Relation between emitted metallic vapor and TSA duration

Figure 12 shows that relation between relative metallic vapor emission of silver and TSA duration before primary arc finished. As shown in Fig. 12, the TSA duration became long if the emitted metallic vapor increased before arc shifting to TSA. The duration of TSA is proportional to amount of emitted metallic vapor.

We focused on the source of the metallic vapor emission of silver. In the case of general arcs, metallic vapor emission from cathode because of the arc current. Figure 13 shows the relation between metallic vapor emission and arc current.

We found that there is strong relation between secondary arc occurrence condition and metallic vapor emission of silver.

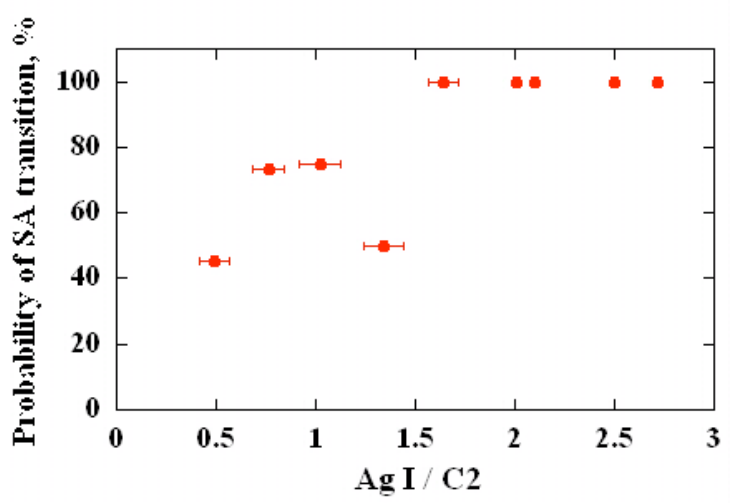

Fig. 11 Probability of TSA transition for arcs at string gap.

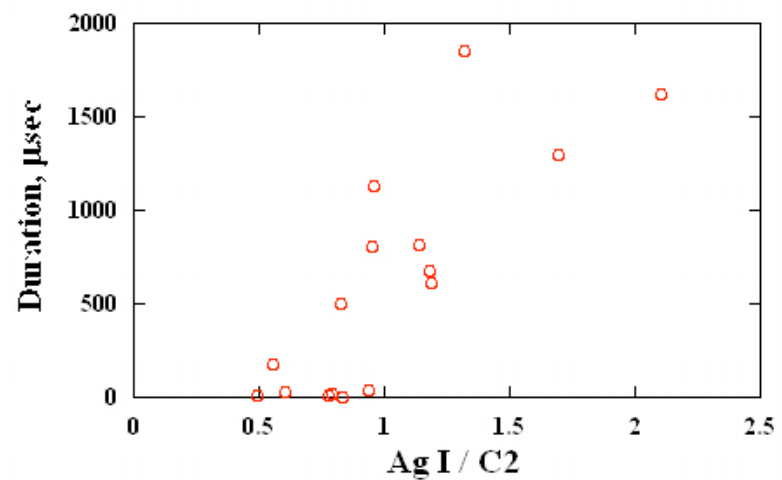

Fig. 12 Relation between amount of emitted metallic vapor and TSA duration.

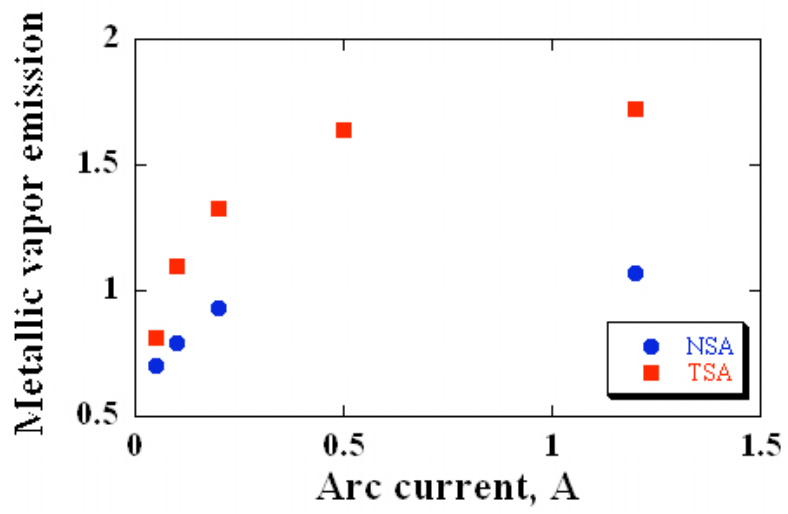

Fig. 13 Relation between arc current and amount of emitted metallic vapor. 
Table 3 PA parameter

\begin{tabular}{|c|c|c|c|c|c|}
\hline \multirow[b]{2}{*}{$\begin{array}{l}\text { Vst } \\
{[\mathrm{V}]}\end{array}$} & \multirow[b]{2}{*}{$\begin{array}{l}\text { Ist } \\
\text { [A] }\end{array}$} & \multirow[b]{2}{*}{$\begin{array}{l}\text { Cext } \\
{[\mathrm{nF}]}\end{array}$} & \multicolumn{3}{|c|}{ Blow-off current } \\
\hline & & & $\begin{array}{c}\text { Peak } \\
{[\mathrm{A}]}\end{array}$ & $\begin{array}{l}\text { Pulse } \\
\text { width } \\
{[\mu \mathrm{sec}]}\end{array}$ & $\begin{array}{c}\text { Charge } \\
{[\mu \mathrm{C}]}\end{array}$ \\
\hline \multirow{3}{*}{70} & \multirow{3}{*}{0.2} & 30 & 20.4 & 18.5 & 150.3 \\
\hline & & 45 & 31.2 & 19.8 & 218.1 \\
\hline & & 65 & 45.6 & 17.8 & 307.3 \\
\hline
\end{tabular}

Table 4 Number of arcs

\begin{tabular}{c|c|c|c|c|c|c}
\hline \hline \multirow{2}{*}{$\begin{array}{c}\text { Vst } \\
{[\mathrm{V}]}\end{array}$} & \multirow{2}{*}{$\begin{array}{c}\text { Ist } \\
{[\mathrm{A}]}\end{array}$} & \multirow{2}{*}{$\begin{array}{c}\text { Cext } \\
{[\mathrm{nF}]}\end{array}$} & \multicolumn{5}{|c}{ Number of arcs } \\
\cline { 3 - 7 } & & 30 & 20 & 14 & 5 & 1 \\
\hline \hline \multirow{3}{*}{70} & \multirow{3}{*}{0.2} & 45 & 20 & 11 & 5 & 4 \\
\cline { 3 - 7 } & & 65 & 11 & 4 & 2 & 5 \\
\hline \hline
\end{tabular}

\subsection{Experimental parameter of $\mathrm{C}_{\text {ext }}$ experiment}

We considered the primary arc energy due to External capacitance $\left(\mathrm{C}_{\text {ext }}\right)$. Table 3 lists the parameter of $\mathrm{PA}$ during this experiment. We carried out the low string current experiment to observe only the effect of PA dimension. In this chapter, we investigate the effect of PA dimension against plasma condition at gap in detail.

\section{SA \& TSA probability for external capacitance}

First, we verified whether probability of secondary arc occurrence changes by PA dimension (the value of external capacitance, $\mathrm{C}_{\mathrm{ext}}$ ) Table 4 shows the the number of arcs at gap with changing $\mathrm{C}_{\text {ext }}$. From this result, we calculated the probability of secondary arc (SA) and

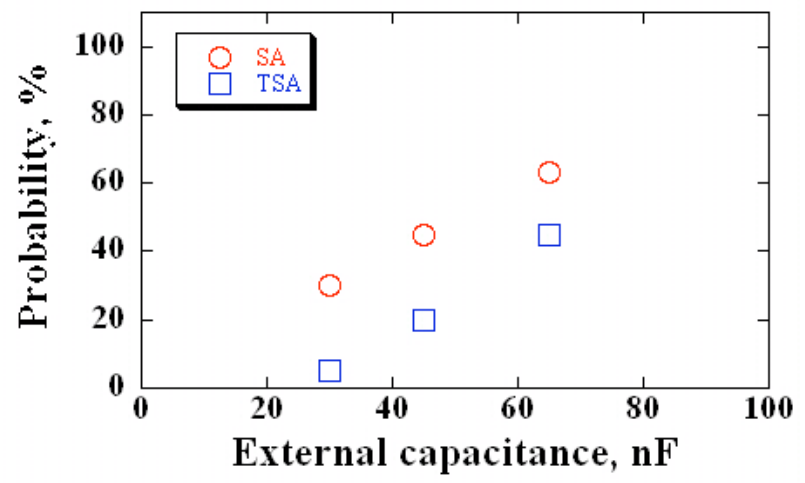

Fig. 14 SA\& TSA probability for external capacitance.

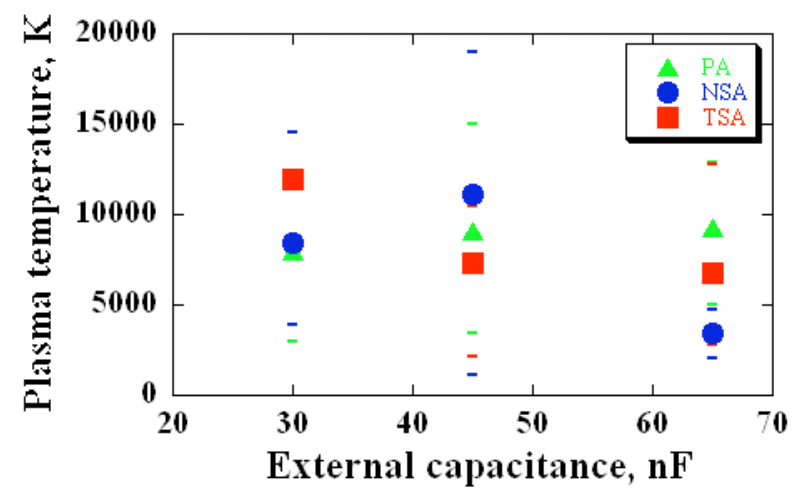

Fig. 15 Plasma temperature for external capacitance.

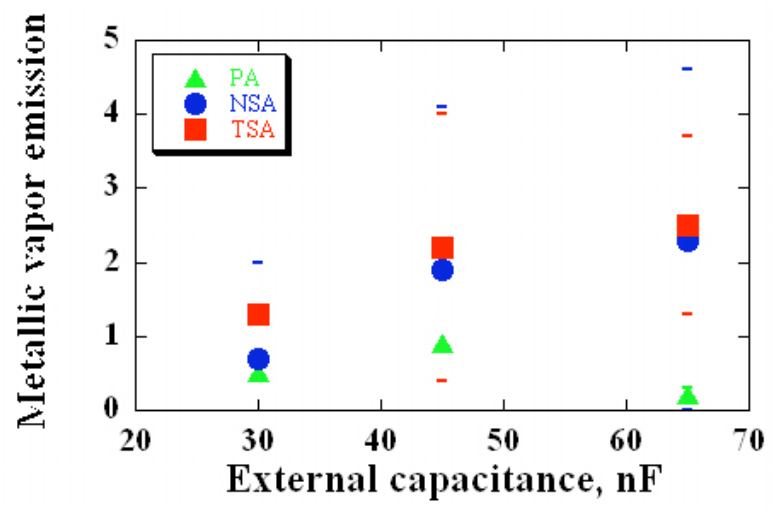

Fig.16 Metallic vapor emission for external capacitance.

temporary sustained arc (TSA) occurrence. Figure 14 shows the calculation result. We found that the bigger PA dimension, the higher probability of SA and TSA occurrence.

\section{Plasma temperature during PA}

We verified whether plasma temperature at gap during PA changes by PA dimension. Figure 15 shows the plasma temperature for PA dimension. The error bar means the minimum and maximum value of plasma temperature. We could not determine plasma temperature depended on the PA dimension.

Metallic vapor emission during PA

We verified whether metallic vapor emission of silver at gap during PA changes by PA dimension. The relation of metallic vapor emission and PA dimension is shown in Fig. 16. The error bar means the minimum and maximum value of metallic vapor emission of silver. This figure shows that the metallic vapor emission depends on PA dimension.

\section{Summary}

In this paper, we identified the material emitted by arc and calculated the arc plasma temperature by means of applying spectroscopy on arc discharge.

We investigated the difference of secondary arcs occurrence condition during secondary arcs. From the spectroscopy results, we found that the vapor of the backside electrode of cell was necessary for shifting from primary arc to the secondary arc. In case of PA dimension changes, the probability of secondary arc and TSA occurrence became high. And plasma temperature was not affected by PA dimension, however the metallic vapor emission of silver was greatly affected. Thus, secondary arc occurrence greatly depends on metallic vapor emission from cathode.

\section{References}

1) Katz, I., Davis V.A; and Snyder, D.B: Mechanism for Spacecraft Charging Initiated Destruction of Solar Arrays in GEO, AIAA 98-1002, 36th Aerospace Sciences Meeting \& Exhibit (1998). 
2) Cho, M., and Fujii, H.: Review on Charging and Discharging Phenomena in Space Environment: Arcing on High Voltage Solar Array and Future Issues, Aeronautical and Space Sciences Japan, 51(2003), pp.139-145.

3) Takahashi, M., Nishimoto, H., Kawakita, S., Cho, M., Nozaki, Y., Fujii, H., Murakami, Y., Ozaki, T., Onodera, N.: ETS-VIII Solar PDL Plasma Interaction Problem Approach", Proceedings of 7th Spacecraft Charging Technology Conference, Noordwijk, Netherlands (2001).

4) NIST Atomic Spectra Database http://physics.nist.gov/cgi-bin/AtData/lines_form

5) Boltzmann Equilibrium Spectrum Program http://view.utsi.edu/besp/

6) Kim, J., Aso, S., Hosoda, S., Cho, M.,: Threshold Conditions to Induce the Sustained Arc on the Solar Array Panel of LEO Satellite, Proceedings of 9th Spacecraft Charging Technology Conference (2005). 